\title{
Landslide Monitoring Network Establishment within Unified Datum and Stability Analysis in the Three Gorges Reservoir Area
}

\author{
Shengxiang Huang, ${ }^{1,2}$ Chenfeng Li, ${ }^{1}$ and Li Luo ${ }^{3}$ \\ ${ }^{1}$ School of Geodesy and Geomatics, Wuhan University, Wuhan 430079, China \\ ${ }^{2}$ Collaborative Innovation Center for Geospatial Technology, Wuhan 430079, China \\ ${ }^{3}$ Liaoning Bureau of Surveying, Mapping and Geoinformation Network Center, Shenyang 110034, China
}

Correspondence should be addressed to Chenfeng Li; cfli@whu.edu.cn

Received 8 June 2017; Accepted 9 August 2017; Published 20 September 2017

Academic Editor: Francesco Dell'Olio

Copyright (c) 2017 Shengxiang Huang et al. This is an open access article distributed under the Creative Commons Attribution License, which permits unrestricted use, distribution, and reproduction in any medium, provided the original work is properly cited.

\begin{abstract}
A landslide monitoring network construction within unified datum which combined fiducial points, working reference points, and monitoring points was intensively studied in the Three Gorges Reservoir area. With special long and narrow geographical location in the area, designing and building monitoring network was vital to the realization of landslide monitoring. To build such a network with high precision, this paper mainly focused on the following four aspects: (1) method of using multiple GPS reference stations to build a unified datum network and subnet adjustment, (2) GPS data processing algorithm with millimeter level, (3) analysis of influence on the adjustment resulting from systematic error of time evolution datum from different GPS observations, and (4) establishment and stability analysis of unified datum. Then, using global test and trial-and-error method to analyze the datum based on the GPS observations (2008 2011) of landslide monitoring network in the area, we concluded that there were moved reference points during the three years of high water impoundment, and the horizontal displacement of moved reference points was more than $4 \mathrm{~cm}$, even up to $79.4 \mathrm{~cm}$. The displacement direction of unstable reference points was inspected with geographical environment at sites, which revealed congruency between them.
\end{abstract}

\section{Introduction}

The total length of the Yangtze River's Three Gorges Reservoir area is 574 kilometers, and the total capacity of the Three Gorges Dam is $3.93 \times 10^{10}$ cubic meters, which is one of the largest dams in the world [1]. Large water storage could easily lead to landslide, collapse, earthquake, and other disasters, putting the residents' lives and property along the reservoir area in danger. For the past few years, GPS technologies have been increasingly frequently applied to monitoring landslide [2-5]. Thus, as one of the monitoring techniques, A-level reference network, B-level working reference network, and C-level deformation network in three levels using GPS were established along the long and narrow reservoir area by related departments of the Three Gorges Reservoir area. Among the network mentioned above, the reference network covers the entire area, which was the landslide monitoring framework [6-8]; the working network was the encryption and expansion based on control network, according to the distribution of the landslide, which provided reference points for landslide monitoring; the deformation monitoring network was laid on the landslide body, jointing with adjacent reference points to monitor the landslide deformation. The establishment of landslide monitoring networks in the Three Gorges Reservoir area has formed a unified entirety, which improved effectively the reliability and sustainability of landslide monitoring.

In 2008, the Three Gorges Reservoir stepped into the stage of the highest level of water storage, and the water level gradually increased from 156 to 175 meters. The rising of water level weakened the stability of reservoir bank, especially the landslides less than 175 meters in elevation. With the action of high water level soaking and periodic flushing, the stability decreased further and easily induced new landslides and collapse events. Therefore, in order to evaluate the reservoir bank stability, we identified 122 key landslides 


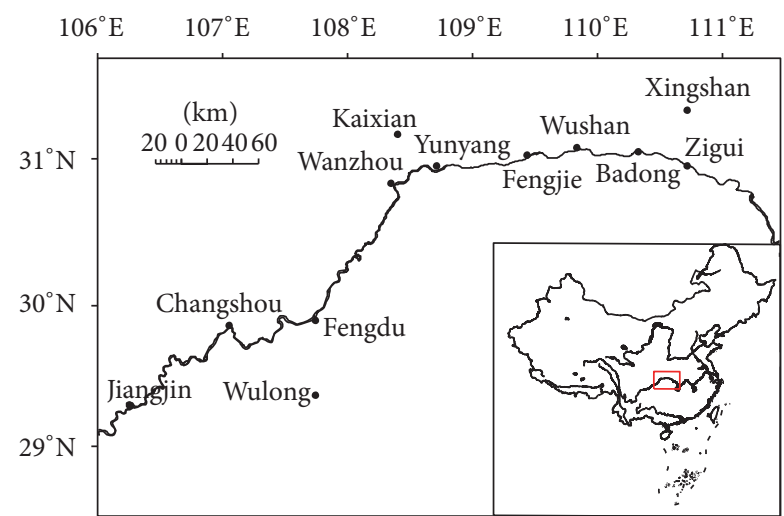

FIGURE 1: The geographical location of the Three Gorges Reservoir area.

and laid working reference points as well as deformation points for landslides monitoring. The meaning of "network construction within unified datum" is the conjunction of working reference points and deformation monitoring points of these newly identified landslides into the fiducial network, using high-precision data processing technology, placing these new landslide monitoring points into the landslide monitoring framework. The landslide monitoring system of the Three Gorges Reservoir area was a basic science project based on the long-term needs of the landslide monitoring after the completion of the landslide monitoring network in the reservoir area. The research on the landslide monitoring GPS network construction within unified datum of the Three Gorges Reservoir area had a long-term significance in both theory and practice.

Based on the characteristics of the GPS system in the Three Gorges Reservoir area, this paper focused on the GPS observation scheme and data processing method and made systematical stability analysis on the control network. The geographical location of the study area is shown in Figure 1.

\section{Characteristics and Analysis of GPS Network Construction within Unified Datum}

2.1. Characteristics of GPS Network Construction within Unified Datum. To build the landslide monitoring GPS network construction within unified datum in the Three Gorges Reservoir area was an extremely special situation. Above all, as the measured area was a long and narrow strip distribution and the roving stations were widely dispersed over a mountainous area, it was inconvenient to go out to conduct research and carry out work. Next in importance, a surprising number of points constituted the control network, where more than 962 points come from B-level network and C-level network and dozens of different types of GPS receiver were involved in the observation. Thirdly, the observation condition got worse because of the reservoir high steep mountain, and more signal would be shielded as result. Last but not least, it was the complex graphical structure that made it important and necessary to establish the GPS network

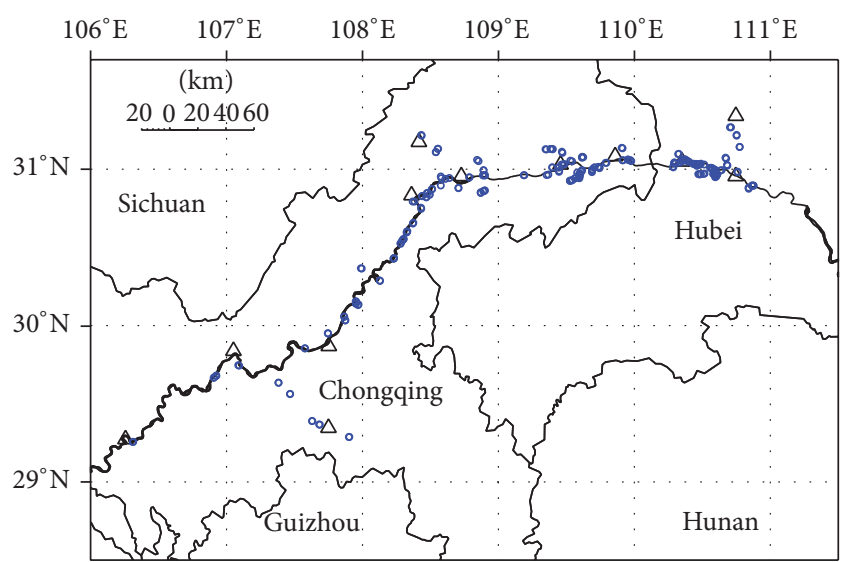
$\triangle$ A-level reference point
- B-level working reference point

FIGURE 2: Point distribution of GPS reference network.

within unified datum, but inevitably the longest baseline was 500 kilometers, and the shortest baseline was 1 kilometer or so; meanwhile the baseline varied greatly in length of the net.

2.2. Problem Analysis. In view of the above characteristics, for the purpose of processing the data with high precision, the following several aspects should be considered in landslide monitoring GPS network construction within unified datum in the Three Gorges Reservoir area.

Firstly, in the aspect of GPS observation scheme, in order to improve the operation efficiency, save operating costs, and ensure the graphics structure strength of GPS network, we adopted the technique of synchronous operation, which could weaken or eliminate the ephemeris error, satellite clock biases, and atmospheric delay to a better situation [9]. The essence of synchronous operation is carrying on the observation simultaneously when we build the GPS reference network and the GPS deformation network; namely, in the process of establishing the GPS reference network, the GPS deformation network is established at the same time. Another thing to notice was setting up multiple reference stations during the all-weather observation to ensure the plurality of reference station. Thus, the working reference points could be observed without waiting for each other, and each working reference point could constitute synchronous graphics with more than three reference points. Meanwhile, all working reference points and the reference points connected to each other to form a reference network. And the deformation monitoring network was based on the working reference points around the landslide mass to make synchronous operation with them. Each deformation point could be combined with at least two working reference points to constitute synchronous graphics. Finally, all deformation points of landslide mass via connection with the working reference points constituted the deformation monitoring network. The distribution of the GPS reference points is shown in Figure 2. 


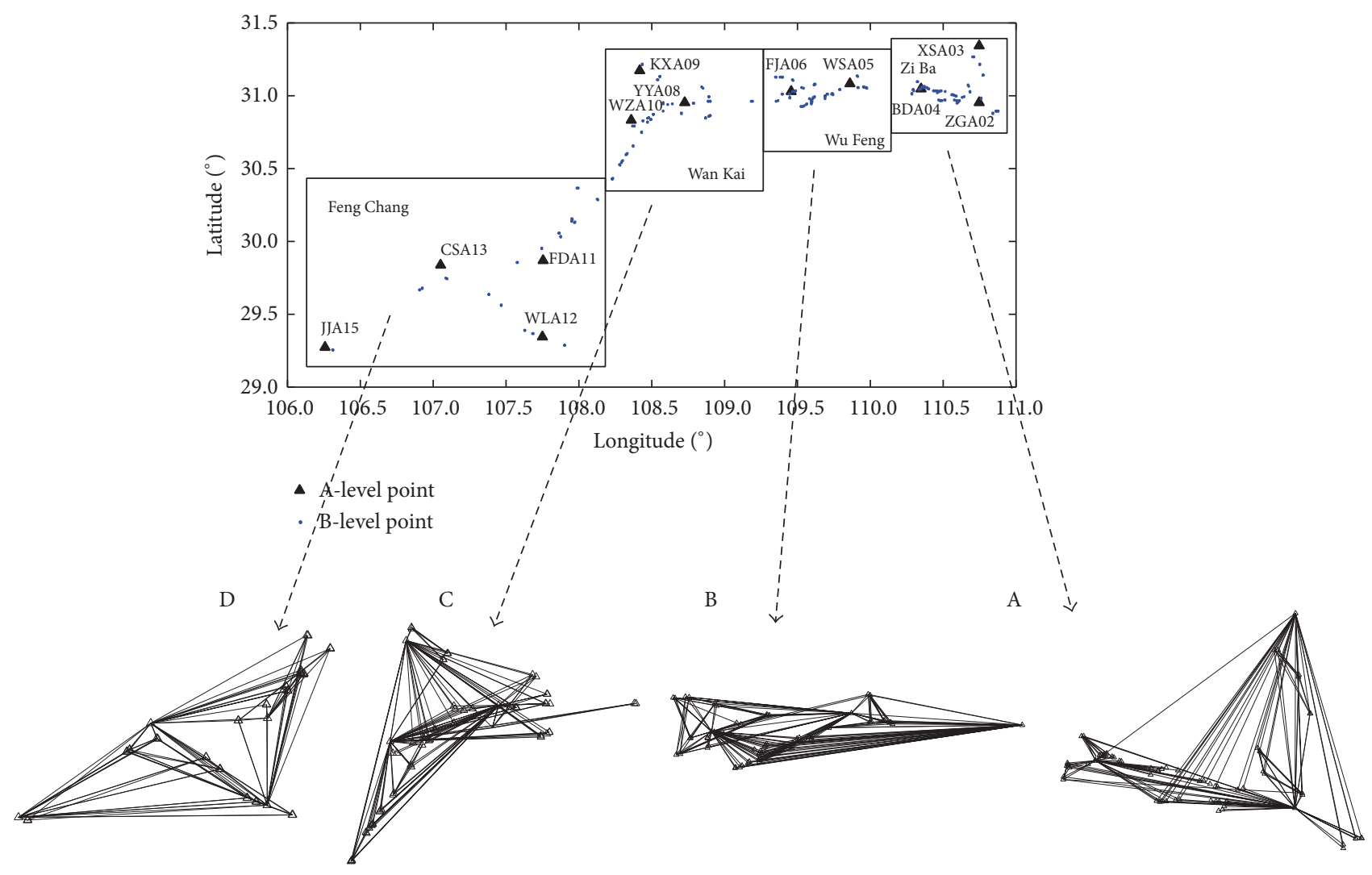

FIgURE 3: Four GPS subnets composed of short baselines (A, B, C, and D).

In the GPS observation process of the project, 57 GPS receivers were actually used and 962 points were observed. If the synchronous graphics expansion of the network method was used, with the B-level network using the net-connection mode and the C-level network using line-connection mode, it would take 54 days to finish the whole observation in theory apart from the time delay due to receivers moving in the measurement area. Adopting the observation scheme studied in this paper, 44 days were used which had increased by nearly $20 \%$ in the operating efficiency and had greatly reduced the difficulty in implementation of the observation.

Secondly, in GPS baseline processing aspect, in the GPS network construction within unified datum, due to the differences in baseline length, different strategies and function models that produced great effect on precision should be focused on before baselines were processed [10]. Thirdly, in terms of GPS network adjustment, when we implemented the adjustment of baselines, gross errors which were much larger than the tolerance under normal observing conditions were eliminated by Delft data-snooping method [11, 12]. In this method, corrections of observations were constructed as a test statistic and whether there was gross error was judged by a certain significance level. Meanwhile, system errors were the datum errors resulting from the systematic difference between observations and reference datum, such as direction datum, measure datum, and time evolution datum. Generally, this type of errors can be removed using functional model with system parameters [13].

\section{Baseline Processing}

3.1. Baseline Processing Strategy. Taking the characteristics of the Three Gorges Reservoir area that was in a long and narrow strip distribution into consideration, the GPS reference network in the reservoir area was divided into four subnetworks with short baselines and one subnetwork with long baselines. And the four short-baseline GPS networks covered the Three Gorges Reservoir area of Zigui-Badong, Fengjie-Wushan, Wanzhou-Yunyang, and Fengdu-Jiangjin, as shown in Figure 3, the serial numbers of which were A, B, $\mathrm{C}$, and D. The long-baseline GPS network was composed of A-level points in the reservoir area and nearby IGS tracking stations, as shown in Figure 4, the number of which was E. According to the length of baseline and the scale of each subnet, length of each baseline from different subnet was made in statistics, and the results were shown in Table 1. When we generated baseline processing strategy, lots of main errors, such as the system error of the satellite orbit error, the ground-reference station coordinate error, the tropospheric delay, and the nontidal atmospheric loading, were studied.

High-precision GPS data processing software needs to be used for the landslide monitoring [14]. GAMIT software developed by MIT (Massachusetts Institute of Technology) and SIO (Scripps Institution of Oceanography) from USA is famous professional GPS data processing software, which helps researchers to compute and analyze GPS data in high precision [15]. The data processing strategies for unified datum in most case are as follows: 


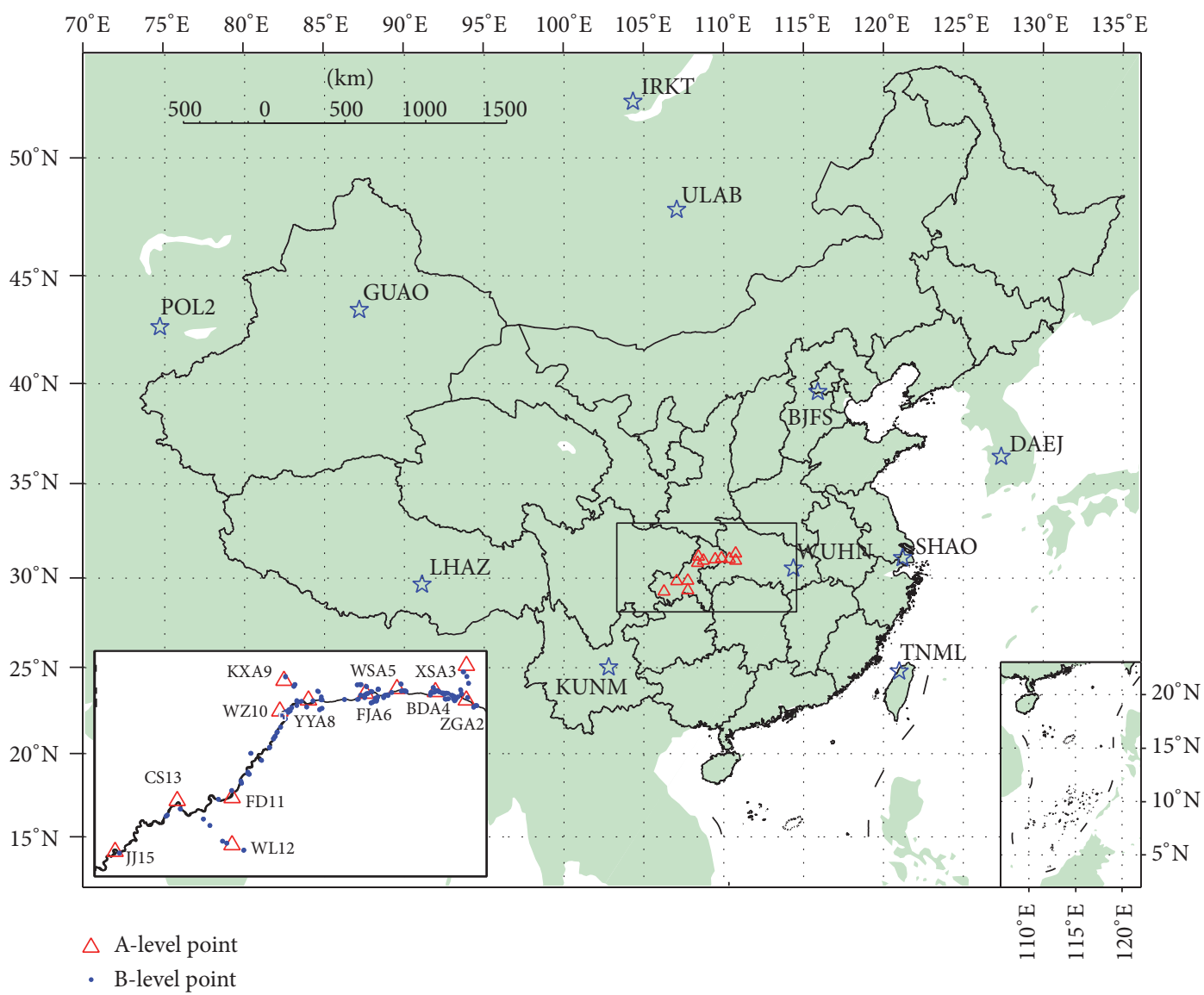

FIGURE 4: Distribution map of landslide monitoring reference points in the Three Gorges Reservoir area and IGS tracking stations.

TABLE 1: Statistical results of baseline length of each subnet.

\begin{tabular}{|c|c|c|c|c|c|c|c|c|c|}
\hline \multirow{2}{*}{ Subnet } & \multirow{2}{*}{ Baseline length } & \multicolumn{2}{|c|}{ Geographical position } & \multicolumn{6}{|c|}{ Baseline length statistics $/ \mathrm{km}$} \\
\hline & & Mean latitude & Mean longitude & $0-50$ & $50-150$ & $150-300$ & $300-500$ & $500-1000$ & $>1000$ \\
\hline A & Short & 31.1 & 110.6 & $98.3 \%$ & $1.7 \%$ & - & - & - & - \\
\hline B & Short & 31.0 & 109.6 & $92.5 \%$ & $7.5 \%$ & - & - & - & - \\
\hline $\mathrm{C}$ & Short & 30.8 & 108.6 & $84.7 \%$ & $15.3 \%$ & - & - & - & - \\
\hline $\mathrm{D}$ & Short & 29.8 & 107.2 & $34.5 \%$ & $65.6 \%$ & - & - & - & - \\
\hline E & Long & 38.6 & 101.1 & $2.0 \%$ & $4.2 \%$ & $2.8 \%$ & $7.1 \%$ & $9.9 \%$ & $74.0 \%$ \\
\hline
\end{tabular}

Observations: original L1 carrier phase observations

Elevation angle cutoff: $10^{\circ}$

Sampling rate: $15 \mathrm{~s}$

Satellite orbit: broadcast ephemeris

Satellite clock biases: double-difference eliminated

Receiver clock biases: double-difference eliminated

Satellite antenna PCO: default values from manufacturer

Satellite antenna PCV: only GPS

Receiver antenna PCO and PCV: not applied

Zenith delay estimation interval: $2 \mathrm{~h}$
By calculating the baseline repeatability, we could assess the results of the baseline processing, and (1) could be used to calculate the repeatability of baseline for each group [16].

$$
\begin{aligned}
R_{c} & =\left[\frac{n /(n-1) \cdot \sum_{i=1}^{n}\left(\left(c_{i}-\bar{c}\right)^{2} / \sigma_{c_{i}}^{2}\right)}{\sum_{i=1}^{n}\left(1 / \sigma_{c_{i}}^{2}\right)}\right]^{1 / 2}, \\
\bar{c} & =\frac{\sum_{i=1}^{n}\left(1 / \sigma_{c_{i}}^{2}\right) \cdot c_{i}}{\sum_{i=1}^{n}\left(1 / \sigma_{c_{i}}^{2}\right)},
\end{aligned}
$$

where $n$ denotes the total number of observing sessions; $c_{i}$ denotes coordinate component or baseline length; $\sigma_{c_{i}}^{2}$ is the variance of $c_{i}$. 
According to the processing strategy in Section 3.1, we have processed the same data in both fixed satellite orbit and loose satellite orbit. If the satellite orbits were regarded as known value, we will fix it and do not estimate it in the parameter estimation. If the orbits of satellites are unknown or cannot be obtained in high precision, they will be regarded as parameters to be estimated with other unknown parameters in function model with certain constraints. That means loose orbit. Table 2 lists the difference in repeatability from two orbits. The results show that if the baseline was less than 300 kilometers, choosing fixed satellite orbit would be better than choosing loose satellite orbit. If the baseline was more than 1000 kilometers, the loose satellite orbit would be recommended. However, if the baseline varied from 300 kilometers to 1000 kilometers, the results would have nothing to do with satellite orbit.

Similarly, by calculating the repeatability, we can conclude that if the baseline was less than 50 kilometers, weak constraint to ground point coordinates would be recommended and strong constraint was better for baseline that was more than 50 kilometers. It was worth mentioning that when we restrain the coordinates of ground-reference station, if it was weak constraint it means the constraint value was 5 meters in each direction, and if it was strong constraint it means the constraint value was 5 centimeters in horizontal direction and 10 centimeters in vertical direction. In addition, the constraint values were recommended by senior experts.

Correction effectiveness of tropospheric delay error in GPS data processing was related to three factors: temperature and atmospheric model, mapping function, and parameters setting.

From computation of different strategies, we could draw a conclusion that if the baseline was less than 150 kilometers, the correction results of GPT (Global Pressure Temperature) model and STP (Standard Temperature Pressure) model would be basically the same. If the baseline varied from 150 kilometers to 1000 kilometers, the correction of GPT model was superior to STP model in horizontal direction, while the correction results of the two models in the vertical direction were consistent. If the baseline was more than 1000 kilometers, the GPT model would be more desirable than STP model.

Based on statistical computation, we have found out that when the satellite elevating angle was more than 15 degrees, adopting the GMF, VMF1, and NMF models of mapping function could get the identical results if the baseline was less than 150 kilometers, whereas the GMF and VMF1 models were superior to the NMF model if the baseline was more than 150 kilometers.

It was easy to set the tropospheric parameters for there were usually two tropospheric parameters setting strategies. One case is that every station was just set a tropospheric parameter if the observing session was short and climate was stable. Another case is that every station in each interval should be set a tropospheric parameter if the observing session was long and climate unstable. The duration of GPS observation for landslide monitoring in the Three Gorges Reservoir Area was generally $8 \sim 10 \mathrm{~h}$, and it was recommended that the troposphere parameter be set to $2 \mathrm{~h}$.
Nontidal atmospheric loading correction was necessary to ensure accuracy and reliability, especially in the vertical component. At the same time, nontidal atmospheric loading correction was related to latitude, and the higher the latitude was, the more effective the correction was.

3.2. Baseline Accuracy Analysis. After baseline computing, 105 one-day results including 1001 groups of repetitive baselines were obtained. The baseline repeatability could be calculated based on (1) and (2) mentioned above; thus, according to (3), the baseline repeatability of the whole network is fitted, and the fitting results are shown in Table 3.

$$
R_{c, k}=a+b \cdot L_{i},
$$

where $R_{c, k}$ indicates the repeatability of baseline $k$; $L_{i}$ is the length of baseline; $a$ and $b$ are the fixed error and proportional error, respectively.

Table 3 shows that the overall resolving accuracy of GPS baseline network is excellent. Relative accuracy in horizontal direction is $10^{-9}$, while that in vertical direction is $10^{-8}$, and the relative accuracy in length is $10^{-9}$.

\section{Network Adjustment}

In view of strip distribution, great difference in length of baseline from the network, various GPS receivers involved to work together, and different systematic error among GPS receivers, the GPS reference network was divided into four subnets from east to west, marked by A, B, C, and D, as shown in Figure 3. The statistics for reference points, working reference points, and one-day results of each subnet are shown in Table 4.

In the first place, nonconstrained adjustments for each subnet were conducted utilizing the observations of independent baseline and their variance-covariance matrix from one-day result of each subnet. The purpose of nonconstrained adjustment for the subnet was to identify whether the subnet contained gross errors or not, as well as to test the existence of systematic errors within stochastic model.

After eliminating the gross errors and correcting the stochastic model for each subnet, it was necessary to study the method of constrained adjustment, which contained two main parts: the method to unify the datum and to eliminate all kinds of system error [17] and suitable adjustment method considering the characteristics of long and narrow strip distribution in the Three Gorges Reservoir area [18].

When building the network with unified datum, it was based on the reference frame ITRF2000 [19] and epoch of 2005.634 where the overall adjustment was conducted for A-level reference points, which was the datum of GPS constrained adjustment in the Three Gorges Reservoir Area. However, the baseline resolving results show that the baseline frame was ITRF2005 [20] and the average observation epoch was 2008.916, resulting in the disunity in observation datum and adjustment datum under the reference frame and epoch.

In order to realize the landslide monitoring within unified datum in the Three Gorges Reservoir area, we have adopted 


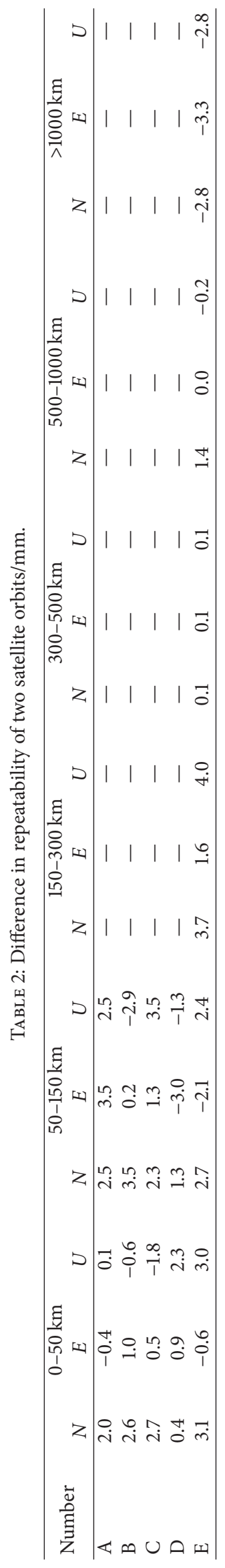




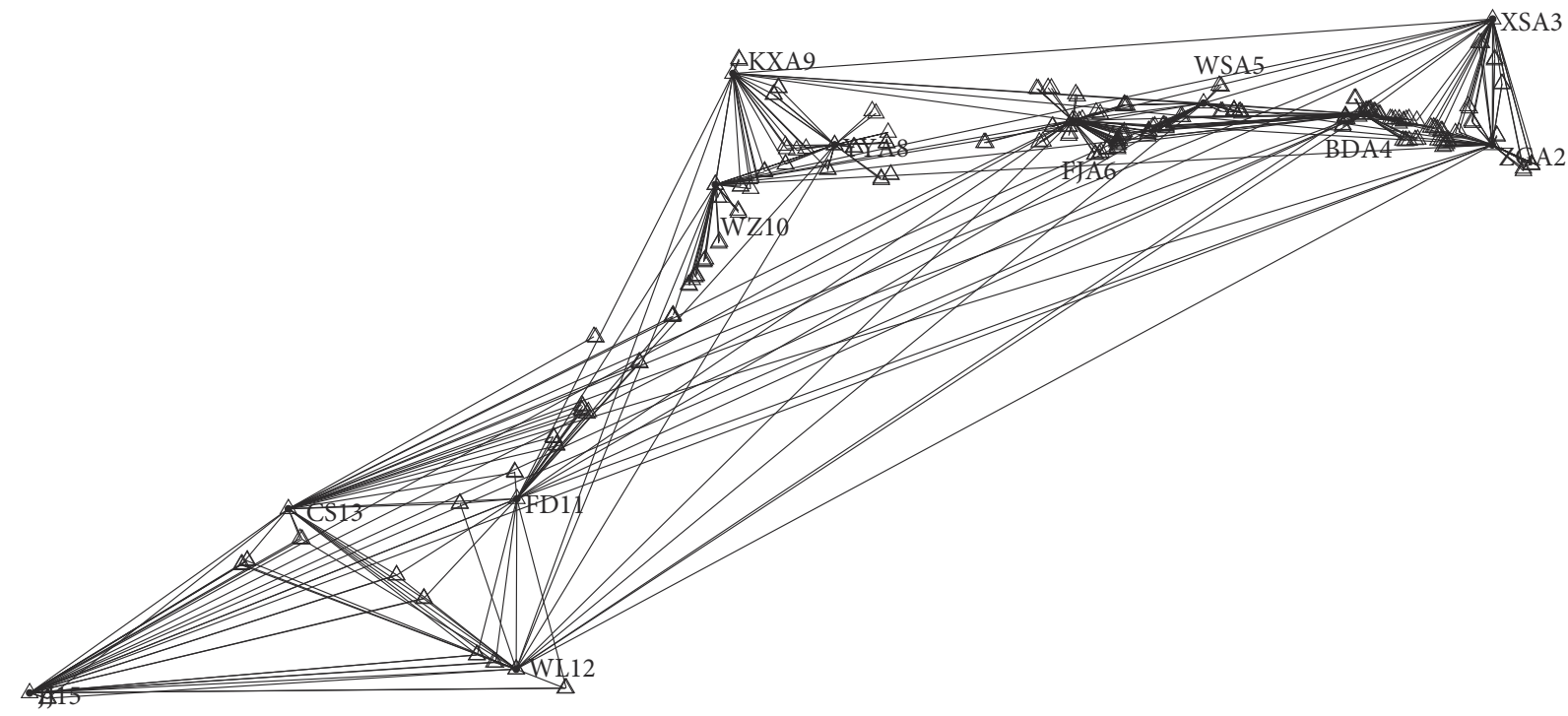

FIGURE 5: Integral adjustment network.

TABLE 3: Internal accord accuracy of the baseline resolving.

\begin{tabular}{lcccc}
\hline Accuracy index & $N$ & $E$ & $U$ & $L$ \\
\hline Fixed error, $a / \mathrm{mm}$ & 2.7 & 2.9 & 5.2 & 2.7 \\
Proportional error, $b / 10^{-8}$ & 0.2 & 0.6 & 3.9 & 0.1 \\
\hline
\end{tabular}

TABLE 4: Information statistics for each subnet.

\begin{tabular}{lccc}
\hline Subnet & Reference points & $\begin{array}{c}\text { Working reference } \\
\text { points }\end{array}$ & One-day solutions \\
\hline A & 3 & 72 & 23 \\
B & 3 & 66 & 21 \\
C & 3 & 61 & 19 \\
D & 4 & 36 & 17 \\
\hline
\end{tabular}

appropriate methods such as measure datum and orientation parameters in the constraint adjustment to achieve the unity of the reference frame. It was equally important to apply the iterative adjustment method to eliminate the system error of the time evolution generated by the difference in the reference epoch [21].

As shown in Figure 5, the integral adjustment involved four subnets, of which the observations were independent baseline vectors from each of the subnets that connected to each other, eliminating the gross error and adjusted by the unit weight variance factor. After extracting the independent baselines in one-day solution from 25 one-day solutions, combining with each subnet baselines, conducting nonconstrained adjustment for the whole network, eliminating the gross error baselines, and adjusting the stochastic model, we realized the integral constrained adjustment for the whole network at the end. The fixed points of the integral adjustment were the 12 A-level GPS reference points of the Three Gorges Reservoir area.

When the zoning adjustment was conducted, the constrained adjustments for $\mathrm{Zi} \mathrm{Ba}$ network, Wu Feng network,

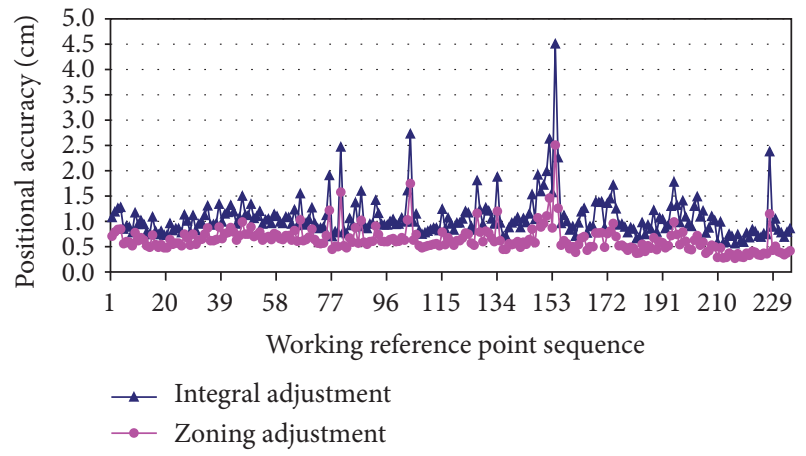

Figure 6: Positional accuracy comparison of integral adjustment and zoning adjustment.

Wan Kai network, and Feng Chang network were done, respectively, and the fixed points were the A-level reference points in every subnet.

By comparison, the adjustment results of the two schemes were basically the same, and the differences in the coordinate components were within $1 \mathrm{~mm}$. However, the difference in positional accuracy was obvious, as shown in Figure 6. In view of accuracy, we decided to adopt the zoning adjustment for GPS reference network.

Next, we briefly analyzed the reliability of network for further analysis. It is confirmed by surveying practice that the GPS net type structure is irrelevant to points' position. For the GPS datum net in this paper and considering the quality index of control network, our concern is the precision and reliability of the net. The reliability could be improved by adding redundant observations. In our works, we adopted the continuously operating reference stations to reinforce the index. According to Baarda's test procedure [11,22], there are internal reliability and external reliability, which need to be analyzed. In view of the complexity of internal and external reliability, we introduced the average reliability to practical 
TABLE 5: Average reliability.

\begin{tabular}{lccc}
\hline Network & Fiducial points & Independent baselines & Reliability \\
\hline Zi Ba & 3 & 160 & 0.9875 \\
Wu Feng & 3 & 139 & 0.9856 \\
Wan Kai & 3 & 132 & 0.9848 \\
Feng Chang & 4 & 108 & 0.9722 \\
Overall/mean & 13 & 539 & 0.9833 \\
\hline
\end{tabular}

work. The average reliability could be computed on the basis of the following equation:

$$
\bar{r}=\frac{r}{n},
$$

where $\bar{r}$ denotes the number of redundant observations and $n$ denotes the number of independent baselines. Based on probability statistics, the larger the value is the higher the reliability is, and the maximum value is 1 . We made statistics to the results of fiducial net reliability analysis as shown in Table 5.

With the statistical Table 5, it is evident that the network is highly reliable.

\section{Stability Analysis}

At present, the research production mentioned above has been applied to the landslide monitoring in the Three Gorges Reservoir area [23]. 2008-2011 was the third stage reservoir impoundment, where the water level increased gradually from $156 \mathrm{~m}$ to $175 \mathrm{~m}$. To ensure the monitoring accuracy, it was necessary to evaluate the datum stability.

In view of that, those 12 fiducial points were far from the reservoir bank and they were set into the bedrock; we preliminarily treated the fiducial points as relatively stable points. In the global reference framework, the horizontal displacement of fiducial points is defined in two parts: rigid body displacement because of the plate movement and local deformation from the plate internal disturbance. Usually, although the former is much larger than the latter, it will not make a difference to the relative position of fiducial points that come from the same plate.

As there were multiperiod observation data, we used the displacement rate to make stability analysis. The highprecision baseline processing software GAMIT/GLOBK was employed to compute the displacement in three steps: (1) to calculate the fiducial points' data with 11 IGS tracking stations nearby and obtain the single-day relaxation solutions of fiducial points, tracking stations and satellite orbit; (2) removing the abnormal solutions after making analysis to the repeatability of the single-day solutions, and then 20 multiday solutions were obtained with three single-day solutions merged into one solution; and (3) calculating the displacement of fiducial points using ITRF2005. Because the WUHN station is in the same plate as those 12 fiducial points, it could be used for testing the results.

In general, the movement of tectonic blocks on a sphere could be described with the rigid motion model as follows [24]:

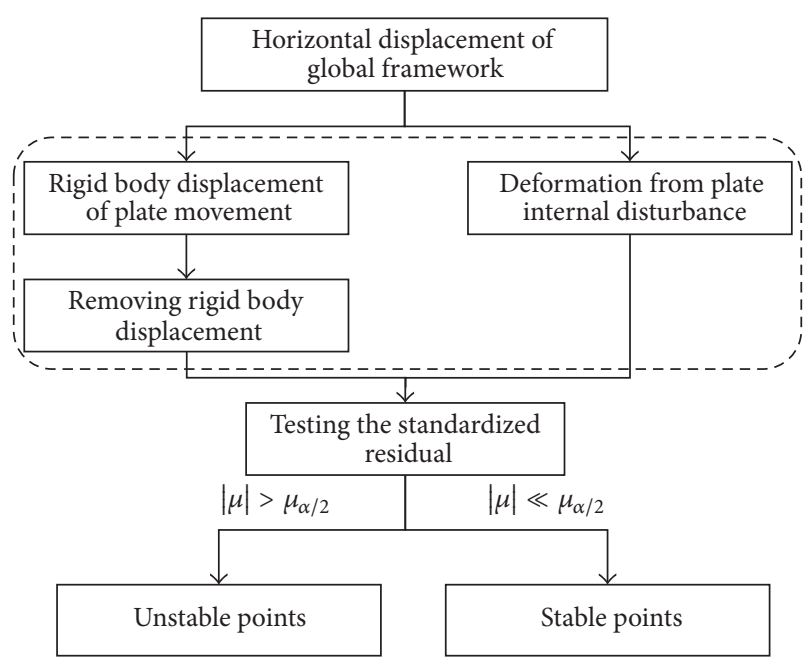

FIGURE 7: Determination flowchart of relatively stable points.

$$
\begin{aligned}
& {\left[\begin{array}{l}
V_{n} \\
V_{e}
\end{array}\right]} \\
& \quad=\left[\begin{array}{ccc}
R \sin L & -R \cos L & 0 \\
-R \sin B \cos L & -R \sin B \sin L & R \cos B
\end{array}\right]\left[\begin{array}{l}
\Omega_{X} \\
\Omega_{Y} \\
\Omega_{Z}
\end{array}\right],
\end{aligned}
$$

where $V_{n}$ and $V_{e}$ are the horizontal rate of station; $\Omega_{X}, \Omega_{Y}$, and $\Omega_{Z}$ are the Euler vector; $R$ is the average earth radius and $B$ and $L$ are the station's latitude and longitude, respectively.

According to the equation above, the displacement rate on the plate of each point could be got. Subsequently, we could make stability analysis to the points by constructing standard normal distribution for further testing:

$$
u=\frac{V_{i}}{\sigma_{0} \sqrt{Q_{V_{i}}}}=\frac{V_{i}}{\sigma_{V_{i}}} .
$$

The flowchart for judging relatively stable points was shown in Figure 7.

In our process, the value of $\alpha$ was set to 0.001 on the basis of experience and practical situation. After displacement calculation with the methods mentioned above, we could work out the displacement for each point. The results were shown in Figure 8. To validate the correctness, we have got the WUHN displacement calculated compared to the official result on the website of IGS. With just small differences in numerical value, the calculation was proven right. As shown in Figure 8(a), under ITRF2005, the displacement rate was about $10 \mathrm{~mm} \cdot \mathrm{a}^{-1}$ in northern direction and $32 \mathrm{~mm} \cdot \mathrm{a}^{-1}$ in eastern direction. This fully explains that there was plate motion. However, from the standardized small displacement shown in Figure 8(b), we could conclude that there was rare movement within plate. And further statistical test was done for making judgment about the stability of the points. All these numerical values indicated that those 12 fiducial points were relatively stable.

In our work, we used two methods to analyze the stability of working reference points: one to compute the 


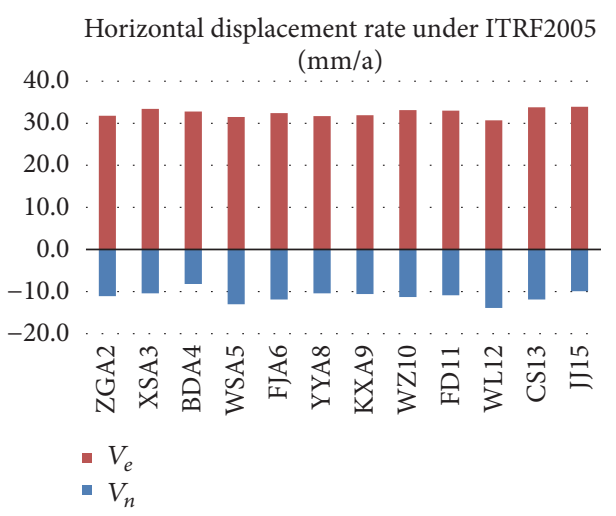

(a)

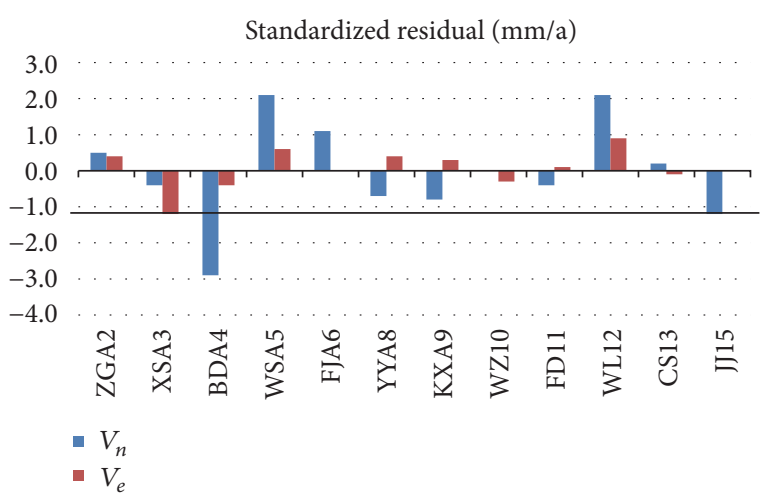

(b)

Figure 8: Displacement of points.

points' displacement and another to check with global test. Displacement calculation of working reference points relative to fiducial points adopted the fixed datum, where the coordinators of fiducial points were fixed among different adjustment of each period. Here we concentrated on the second method.

Global test and data snooping are the most frequently used postadjustment data screening techniques [25]. We have used global test and trial-and-error method to analyze the stability of working reference points [26].

The global test was also known as the method of mean gap, which was put forward by Pelzer from Germany in 1971. The basic thought of the method is to check the congruency of two-period control network in the first place and if the check passes, then all the points are stable; otherwise, the unstable points should be found out by trial-and-error method. When we use the trial-and-error method, each point is taken out successively; then the reduction of the two-period control network in congruency is computed and the corresponding point is the unstable one. Repeat the above process after getting rid of the unstable point until the congruency check is passed. For example, we analyze the network stability of series 1 and $j$. After rank defect-free network adjustment is conducted, according to each period's result, unit weight variance could be acquired by the following equation:

$$
\begin{aligned}
& \mu_{1}^{2}=\frac{\left(V^{T} P V\right)^{1}}{f_{1}}, \\
& \mu_{j}^{2}=\frac{\left(V^{T} P V\right)^{j}}{f_{j}} .
\end{aligned}
$$

Normally, the two-period results are equal in precision (could be certified if necessary). So $\mu_{1}^{2}$ could be combined with $\mu_{1}^{2}$ to calculate a conjunct unit weight variance by the following equation:

$$
\mu^{2}=\frac{\left(V^{T} P V\right)^{1}+\left(V^{T} P V\right)^{j}}{f},
$$

where $f=f_{1}+f_{1}$.
If the hypothesis that the points did not change is made, then, using the coordinate difference $\Delta X$ between the two periods, another variance could be obtained by the following equation:

$$
\theta^{2}=\frac{\Delta X^{T} P_{\Delta X} \Delta X}{f_{\Delta X}},
$$

where $P_{\Delta X}=Q_{\Delta X}^{+}=\left(Q_{x_{j}}+Q_{x_{1}}\right)^{+}$and $f_{\Delta X}$ is the number of independent $\Delta X$.

It turns out that $\mu^{2}$ and $\theta^{2}$ are statistically independent. By $F$-test, the statistic could be acquired:

$$
F=\frac{\theta^{2}}{\mu^{2}} .
$$

Under null hypothesis $H_{0}$ that the points did not change, the statistic $F$ obeys the distribution that the degrees of freedom are $f_{\Delta X}$ and $f$, and the equation

$$
P\left(F>F_{1-\alpha}\left(f_{\Delta X}, f\right) \mid H_{0}\right)=\alpha
$$

could be used to verify the points' stability. The confidence level $\alpha$ is usually equal to 0.05 or 0.01 , and the concrete value $F_{1-\alpha}\left(f_{\Delta X}, f\right)$ could be in the $F$-test table according to $\alpha, f_{\Delta X}$, and $f$. In this paper, we used the confidence level of 0.01 . The confidence level was usually based on the nature of the question and experience. If the null hypothesis should not be refused readily, smaller value should be used. In our computation, we chose 0.01 for overall testing procedure. The test statistic of global test reflects the global congruency of two-period control network. In other words, based on probability and statistics, the probability of error decreases with decreasing significance level.

If the statistic $F$ is less than corresponding value, then as there are no enough evidences to doubt the null hypothesis we should accept that the points are stable and the stability analysis is finished.

If the statistic $F$ is more than corresponding value, then the null hypothesis should be refused, which means that there are unstable points. In this case, we cannot judge which point 
or points have changed. However, there is a way derived from the mean gap to search the unstable points. In this method, the points are divided into two groups, and they are stable points $(F)$ and unstable points $(M)$. There may be both stable points and unstable points in $F$. And now we want to ensure if there are unstable points in $F$ by the test of network congruency. $M$ :

Express $\Delta X$ and $P_{\Delta X}$ with block matrix according to $F$ and

$$
\begin{aligned}
\Delta X^{T} & =\left(\begin{array}{ll}
\Delta X_{F}^{T} & \Delta X_{M}^{T}
\end{array}\right), \\
P_{\Delta X} & =\left(\begin{array}{ll}
P_{F F} & P_{F M} \\
P_{M F} & P_{M M}
\end{array}\right) .
\end{aligned}
$$

Because $\Delta X_{F}$ is correlated to $\Delta X_{M}$, which means $P_{F M}=$ $P_{M F}^{T} \neq 0, \Delta X_{F}^{T} P_{F F} \Delta X_{F}$ cannot reflect the network congruency in $F$ which is influenced by $M$. In order to obtain the network congruency index of $F$, the following transformation is done:

$$
\begin{aligned}
\overline{\Delta X_{M}} & =\Delta X_{M}+P_{M M}^{-1} P_{M F} \Delta X_{F}, \\
\overline{P_{F F}} & =P_{F F}-P_{F M} P_{M M}^{-1} P_{M F} .
\end{aligned}
$$

So the equation

$$
\Delta X^{T} P_{\Delta X} \Delta X=\Delta X_{F}^{T} \overline{P_{F F}} \Delta X_{F}+\overline{\Delta X_{M}^{T}} P_{M M} \overline{\Delta X_{M}}
$$

could be obtained. And $\Delta X^{T} P_{\Delta X} \Delta X$ is divided into two independent items, in which the first means the network congruency of $F$.

Assuming that

$$
\theta_{F}^{2}=\frac{\Delta X_{F}^{T} \overline{P_{F F}} \Delta X_{F}}{f_{F}},
$$

the stability test statistic of $F$ is

$$
F_{1}=\frac{\theta_{F}^{2}}{\mu^{2}} .
$$

If $F_{1}<F\left(f_{F}, f_{1}+f_{j}\right)$, then it means that there are unstable points in $F$.

Otherwise, if $F_{1}>F\left(f_{F}, f_{1}+f_{j}\right)$, it means that there are no unstable points in $F$.

All unstable points can be found in turn by the statistical test and the following searching method.

Supposing that there are unstable points based on global test, there is at least one unstable point. Although we do not know how many unstable points there are, we can firstly seek out the first one and then test the stability of the remaining points by global test. Find out the unstable points until there are no unstable points.

When we search the first unstable point, every point should be checked. All points would be divided into two groups when the point $i$ is tested. One is $i$ as unstable group and the other is remaining points of the network as stable group. Assuming that there are $t(t>2)$ points, the divided groups are

$$
\begin{gathered}
F_{i}: 1,2, \ldots, i-1, i+1, \ldots, t, \\
M_{i}: i
\end{gathered}
$$

computing $\overline{\Delta X_{M_{i}}^{T}} P_{M_{i} M_{i}} \overline{\Delta X_{M_{i}}}$, which is regarded as the index to judge the stability of $i$. Each point must be divided and calculated to acquire the $t$ corresponding index. And the point with the largest index is the potential unstable point.

$$
\begin{array}{r}
\overline{\Delta X_{M_{j}}^{T}} P_{M_{j} M_{j}} \overline{\Delta X_{M_{j}}}=\max \left(\overline{\Delta X_{M_{i}}^{T}} P_{M_{i} M_{i}} \overline{\Delta X_{M_{i}}}\right) \\
(i=1,2, \ldots, t) .
\end{array}
$$

The point $j$ is the potential unstable one.

After seeking out the potential unstable point $j$, check the congruency of the network constructed by the remaining points. The stability analysis is finished if the remaining points are tested to be stable. Otherwise, continue searching the second potential unstable point in the same way as searching the first one. Thus, the point $i$ could be found out from the remaining $t-1$ points to form the unstable group with $j$ point and the others form the stable group:

$$
\begin{gathered}
F_{i j}: 1,2, \ldots, i-1, i+1, \ldots, j-1, j+1, \ldots, t, \\
M_{i j}: i, j .
\end{gathered}
$$

Similarly, $\overline{\Delta X_{M_{i}}^{T}} P_{M_{i} M_{i}} \overline{\Delta X_{M_{i}}}$ could be calculated. In order to find out the second potential unstable point, $t-1$ times dividing and computing need to be done, and take the point $l$ as the second potential unstable point corresponding to the following equation:

$$
\begin{aligned}
\overline{\Delta X_{M_{l j}}^{T}} P_{M_{l j} M_{l j}} \overline{\Delta X_{M_{l j}}}=\max \left(\overline{\Delta X_{M_{i j}}^{T}} P_{M_{i j} M_{i j}} \overline{\Delta X_{M_{i j}}}\right) \\
(i=1, \ldots, j-1, j+1, \ldots, t) ;
\end{aligned}
$$

then check the network congruency of the remaining $t-2$ points.

Repeat this process until the remaining points were tested to be stable.

The observation data from working reference points have been calculated with the two methods described above and we got the same results about the stability of points. There were unstable reference points during the period of GPS observation data in each subnet and the detailed statistical results were shown in Table 5, and the distribution of the unstable reference points was shown in Figure 9.

As you can see in Table 6 and Figure 9, we can conclude that, (1) in 2008 to 2011, there were 12 unstable reference points in the reservoir area, up to about $5 \%$ of the total points; (2) the distribution of the unstable reference points was "east more and west less," and the points were relatively concentrated on Fengjie-Wushan area; (3) the horizontal displacement of the unstable reference points was more than 4 centimeters, and the maximum point even moved up to 79.4 centimeters; (4) there was obvious congruency in the displacement direction of the unstable reference points; (5) for the points of instability, the displacement was proportional to time in the long term.

In addition, we inspected the displacement direction of unstable working reference points on the spot, and we found that the displacement direction of these points was 


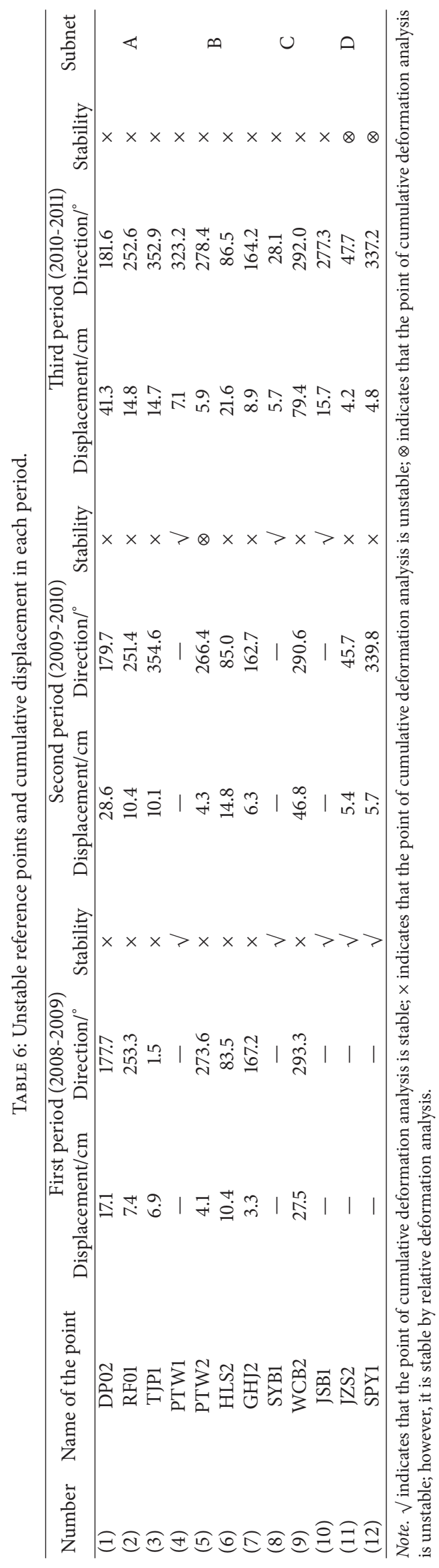




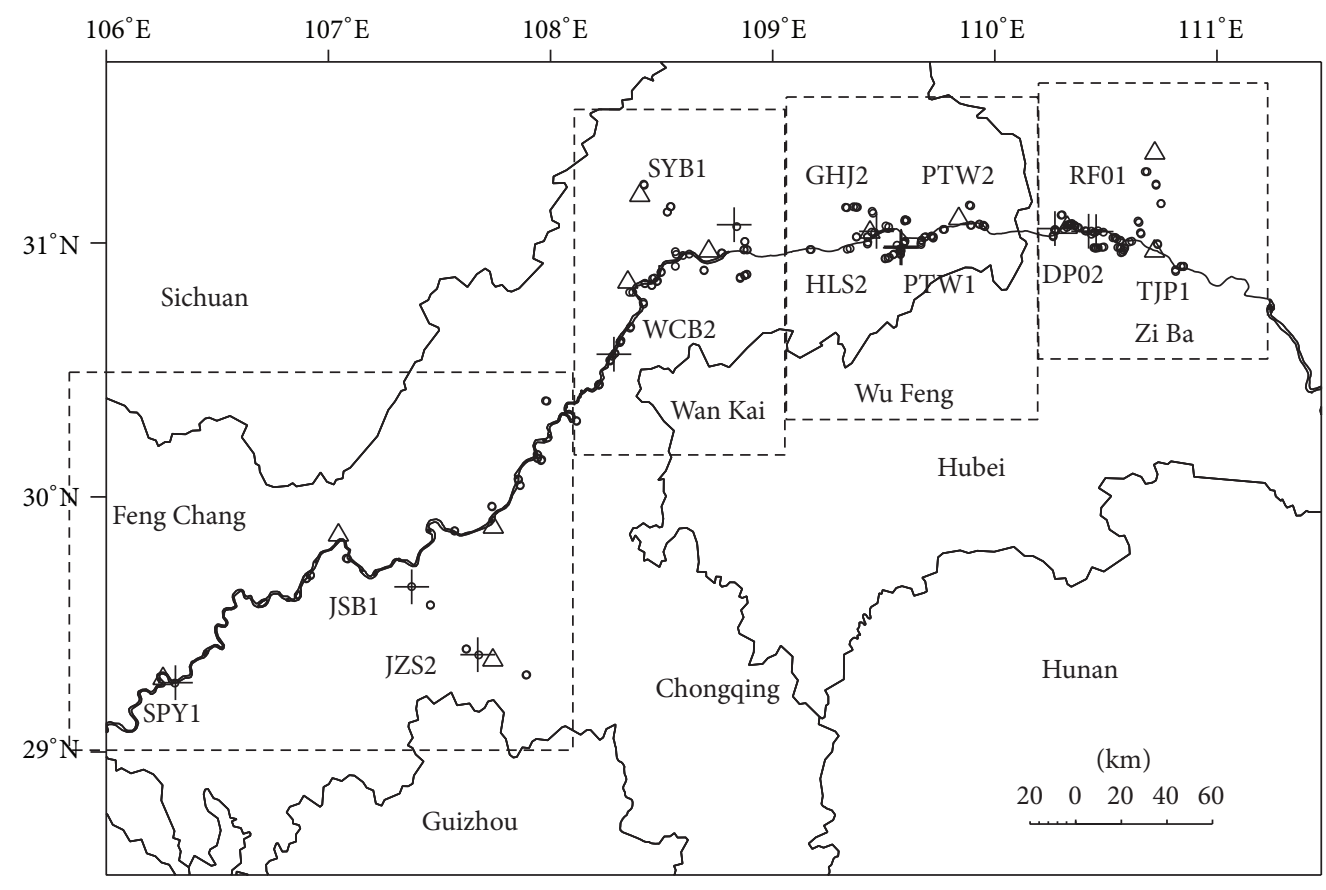

$\triangle$ Stable control point
- Stable reference point
+ Unstable reference point

FIGURE 9: Distribution of unstable reference points.

pointing to the sliding direction, which was consistent with deformation trend based on geographical environment.

\section{Conclusions}

After three years of high water level storage of the Three Gorges Reservoir, it turned out that the landslide monitoring datum network as a whole was stable, which also reflected that there were unstable working reference points around the reservoir, and the horizontal displacement was up to 79.4 centimeters. Experimental results indicated that with our method the detail displacement of unstable points could be computed, and the result was congruent with the onsite calibration. If these unstable working reference points were regarded as the reference of landslide deformation, wrong judgment for landslide deformation evolution behavior would be made, so regular stability analysis for monitoring datum was an indispensable part in the course of landslide monitoring in the reservoir area and it has turned out to be true.

The unique layout form of the landslide monitoring network in the Three Gorges Reservoir area made the stability analysis on the reference points special. In this paper, a set of suitable methods was used to study the landslide monitoring in special environment and the method possesses important guiding significance to specific operation.

\section{Conflicts of Interest}

The authors declare that there are no conflicts of interest.

\section{Authors' Contributions}

Shengxiang Huang devised the research and Li Luo and Chenfeng Li designed methods and experiments. All authors wrote the paper. All authors have read and approved the final manuscript.

\section{Acknowledgments}

This paper was supported by the National Natural Science Foundation of China (Grant no. 41274020). The authors are grateful to the Headquarters of Prevention and Control for Geohazard for the GPS observation data in the Three Gorges Reservoir region.

\section{References}

[1] R. L. Du, X. J. Qiao, Q. Wang, S. F. Qi, and X. Z. You, "Deformation in the Three Gorges Reservoir after the first impoundment determined by GPS measurements," Progress in Natural Science, vol. 14, no. 9, pp. 1006-1011, 2004.

[2] J. A. Gili, J. Corominas, and J. Rius, "Using Global Positioning System techniques in landslide monitoring," Engineering Geology, vol. 55, no. 3, pp. 167-192, 2000.

[3] J. P. Malet, O. Maquaire, and E. Calais, "The use of Global Positioning System techniques for the continuous monitoring of landslides: application to the Super-Sauze earthflow (Alpesde-Haute-Provence)," Geomorphology, vol. 43, no. 1-2, pp. 33$54,2002$. 
[4] F. Tagliavini, M. Mantovani, G. Marcato, A. Pasuto, and S. Silvano, "Validation of landslide hazard assessment by means of GPS monitoring technique-a case study in the Dolomites (Eastern Alps, Italy)," Natural Hazards \& Earth System Science, vol. 7, no. 1, pp. 185-193, 2007.

[5] G. Wang, "GPS landslide monitoring: single base vs. network solutions - a case study based on the Puerto Rico and Virgin Islands permanent GPS network," Journal of Geodetic Science, vol. 1, no. 3, pp. 191-203, 2011.

[6] Z. Y. Shao, J. N. Liu, W. P. Jiang, and Y. X. Li, "Piecewise linear estimation of tropospheric refraction bias in precise positioning of GPS," Crustal Deformation and Earthquake, vol. 18, no. 3, pp. 14-18, 1998.

[7] D. D. Mccarthy and G. Petit, "IERS conventions," Highlights of Astronomy, vol. 13, 2004.

[8] S. Böckmann, T. Artz, and A. Nothnagel, "VLBI terrestrial reference frame contributions to ITRF2008," Journal of Geodesy, vol. 84, no. 3, pp. 201-219, 2010.

[9] Z. H. Li and J. S. Huang, GPS Surveying and Data Processing, Wuhan University Press, Wuhan, China, 3rd edition, 2016.

[10] J. N. Liu, Y. B. Yao, and C. Shi, "Method for establishing the speed field model of crustal movement in China," Geomatics and Information Science of Wuhan University, vol. 27, no. 4, pp. 331-335, 2002.

[11] W. Baarda, A Testing Procedure for Use in Geodetic Networks, vol. 2, no. 5 of Geodesy, Netherlands Geodetic Commission, Delft, The Netherlands, 1968.

[12] P. J. G. Teunissen, Testing Theory: An Introduction, Delft University Press, Delft, The Netherlands, 2000.

[13] Surveying Adjustment Department of School of Geodesy and Geomatics of Wuhan University, Error Theory and Foundation of Surveying Adjustment, Wuhan University Press, Wuhan, China, 3rd edition, 2014.

[14] Z. H. Li and X. H. Zhang, New Techniques and Precise Data Processing Methods of Satellite Navigation and Positioning, Wuhan University Press, Wuhan, China, 2009.

[15] Y. Bock, S. A. Gourevitch, C. C. I. Counselman et al., "Interferometric analysis of GPS phase observations," Manuscripta Geodaetica, vol. 11, no. 4, pp. 282-288, 1986.

[16] T. H. Dixon, G. Gonzalez, S. M. Lichten, and E. Katsigris, "First epoch geodetic measurements with the Global Positioning System across the northern Caribbean plate boundary zone," Journal of Geophysical Research, vol. 96, no. 2, pp. 2397-2415, 1991.

[17] S. X. Huang, "Stability analysis for deformation monitoring network," Journal of Geomatics, no. 3, pp. 16-19, 2001.

[18] Y. X. Yang, "Crustal deformation corrections for the combined adjustment of geodetic network with multi-epoch observations," Geomatic Science and Engineering, vol. 23, no. 1, pp. 4-7, 2003.

[19] Z. Altamimi, P. Sillard, and C. Boucher, "The impact of a no-netrotation condition on ITRF2000," Geophysical Research Letters, vol. 30, no. 2, pp. 36-1-36-4, 2003.

[20] Z. Altamimi, X. Collilieux, J. Legrand, B. Garayt, and C. Boucher, "ITRF2005: a new release of the international terrestrial reference frame based on time series of station positions and earth orientation parameters," Journal of Geophysical Research, vol. 112, no. 9, pp. 83-104, 2007.

[21] Q. Zhang, G.-W. Huang, X.-G. Ding, L. Wang, and C.-Y. Zhao, "Research and realization of high-precision GPS datum, considering plate movement, stability and system errors," Chinese Journal of Geophysics, vol. 52, no. 12, pp. 3158-3165, 2009.
[22] P. J. G. Teunissen, "Quality control in geodetic networks," in Optimization and Design of Geodetic Networks, E. Grafarend and F. Sanso, Eds., pp. 526-547, Springer, Berlin, Germany, 1985.

[23] S. X. Huang and L. Luo, "Stability analysis and results of the landslide monitoring datum in the Three Gorges Reservoir area," Geomatics and Information Science of Wuhan University, vol. 39, no. 3, pp. 367-372, 2014.

[24] C. DeMets, R. G. Gordon, D. F. Argus, and S. Stein, "Current plate motions," Geophysical Journal International, vol. 101, no. 2, pp. 425-478, 1990.

[25] S. L. Kuang, Geodetic Network Analysis and Optimal Design: Concepts and Application, Ann Arbor Press, Chelsea, Mich, USA, 1996.

[26] H. Pelzer, Zur Analyse Geodatischer Deformationsmessungen, vol. 164 of Deutsche Geodätische Kommission, Verlag Der Bayer Akad D Wiss München, München, Germany, 1971. 


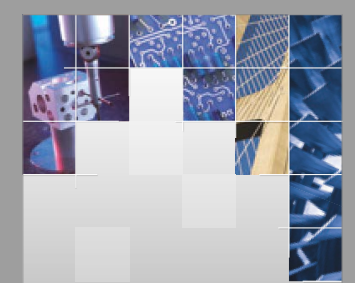

\section{Enfincering}
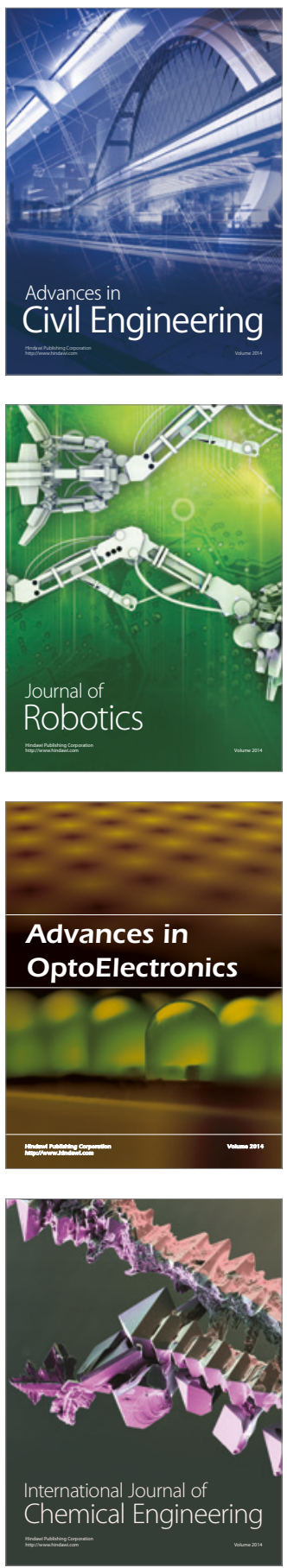

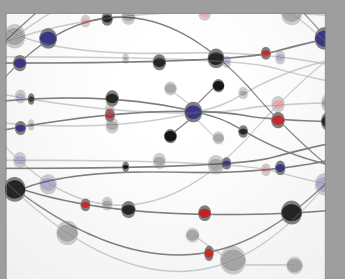

The Scientific World Journal

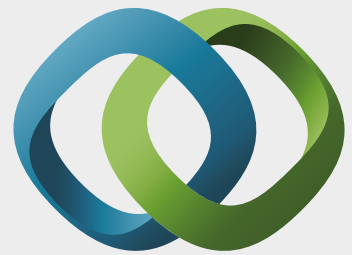

\section{Hindawi}

Submit your manuscripts at

https://www.hindawi.com
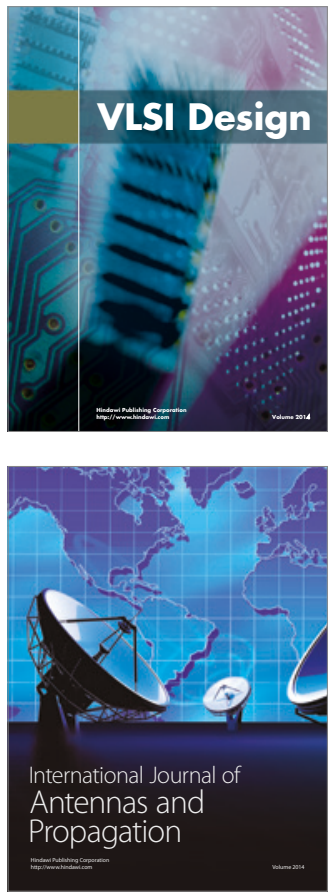

\section{Rotating}

Machinery
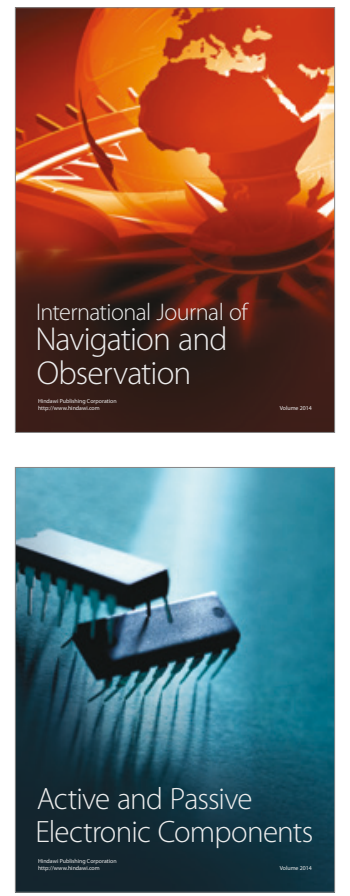
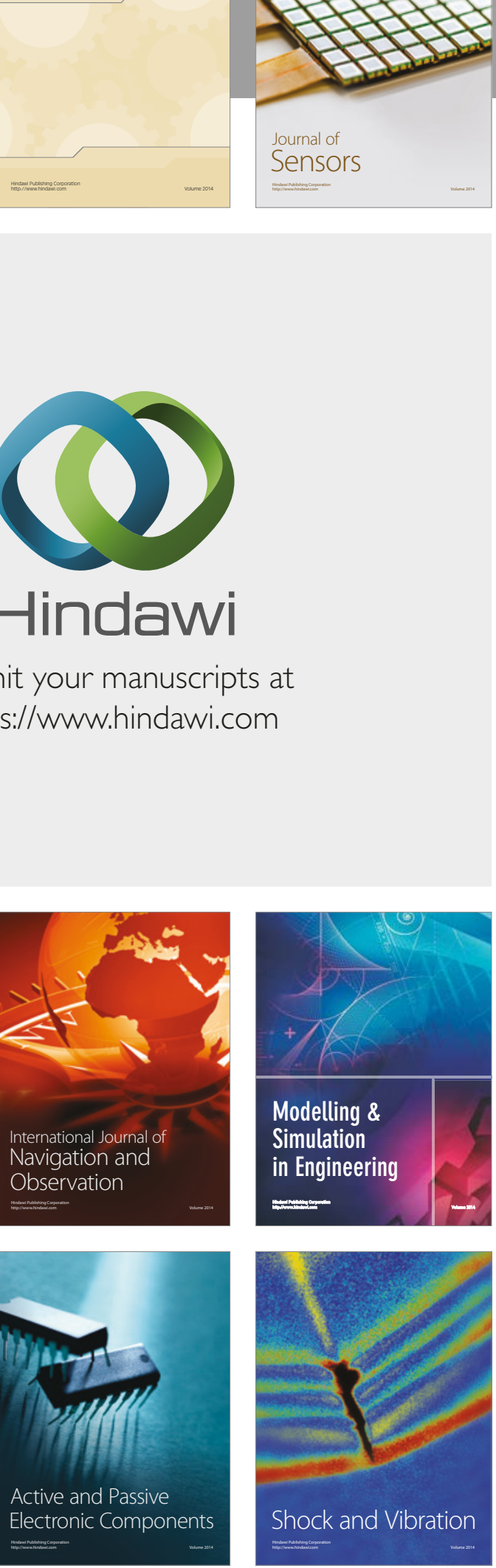
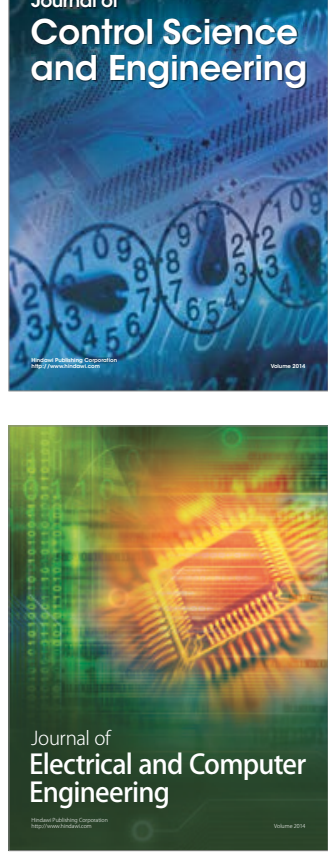

Distributed

Journal of

Control Science

and Engineering
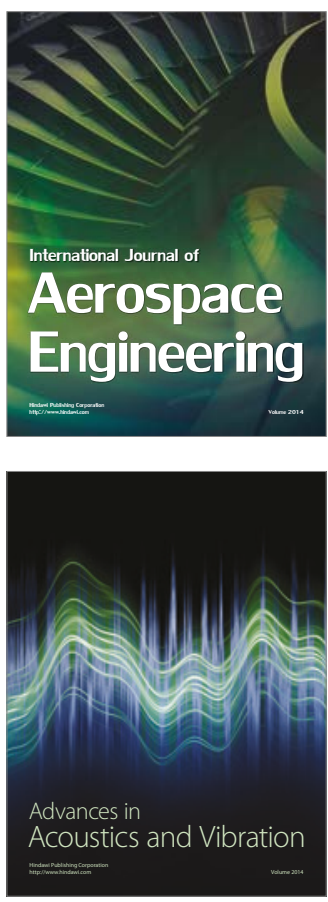

Sensor Networks 